\title{
Favorable safety experience of local dental anesthesia in ICD recipients with channelopathies
}

\author{
Ana Carolina Oliveira ${ }^{1}$, Itamara Neves ${ }^{1}$, Luciana Sacilotto ${ }^{1}$, Natália Olivetti ${ }^{1}$, Savia \\ Bueno $^{1}$, Gabrielle Pessente ${ }^{1}$, Marcela Paul ${ }^{1}$, Tânia Montano ${ }^{1}$, Cíntia Carvalho ${ }^{1}$, Cesar \\ Grupi $^{1}$, Sílvio Barbosa ${ }^{1}$, Carlos Alberto Pastore ${ }^{1}$, Nelson Samesima ${ }^{1}$, Tan $\mathrm{Wu}^{1}$, Denise \\ Hachul $^{1}$, Mauricio Scanavacca ${ }^{1}$, Ricardo Neves ${ }^{1}$, and Francisco Darrieux ${ }^{1}$ \\ ${ }^{1}$ Universidade de Sao Paulo Faculdade de Medicina Hospital das Clinicas Instituto do \\ Coracao
}

July 16, 2020

\begin{abstract}
Introduction - Dental anesthetic management in ICD recipients with CCh can be challenging due to the potential risk of life-threatening arrhythmias and appropriate ICD therapies during procedural time. We assessed the hypothesis that the use of local dental anesthesia with $2 \%$ lidocaine with 1:100.000 epinephrine or without a vasoconstrictor can be safe in selected ICD and CCh patients, not resulting in life-threatening events. Methods and Results - Restorative dental treatment under local dental anesthesia was made in two sessions, with a wash-out period of 7 days (cross-over trial), conducting with a $28 \mathrm{~h}$ - Holter monitoring, and 12-lead electrocardiography, digital sphygmomanometry, and anxiety scale assessments in 3 time periods. Ventricular/supraventricular arrhythmias frequency, device shocks, corrected QT interval and dynamic changes in right precordial leads in $\mathrm{BrS}$ were also analyzed. All patients were in stable condition with no recent events before the dental care. Twenty-four consecutive procedures were performed in 12 patients (9 women, 3 men) with CCh and ICD: 7 (58.3\%) had LQTS, $4(33.3 \%)$ had BrS and 1 (8.3\%) had CPVT. Holter analysis did not demonstrated increased heart rate or sustained arrhythmias. Blood pressure, electrocardiographic changes and anxiety measurement showed no statistically significant differences. No lifethreatening events occurred during dental treatment, regardless the type of anesthesia. Conclusions - Lidocaine administration, with or without epinephrine, can be safely used in selected CCh-ICD patients without life-threatening events, as long as the protocol is followed. These preliminary findings need to be confirmed in a larger population with ICD and CCh.
\end{abstract}

\section{INTRODUCTION}

Implantable cardioverter defibrillator (ICD) detects ventricular tachycardia (VT) and ventricular fibrillation (VF) and delivers therapy in the form of overdrive antitachycardia pacing (ATP), low-energy cardioversion, and high-energy defibrillation ${ }^{1}$. It is indicated for patients at high risk for malignant ventricular tachyarrhythmias (primary prophylaxis) and in patients who have survived from a malignant ventricular tachycardia (secondary prophylaxis) ${ }^{2}$.

ICD remains an effective therapeutic option to prevent sudden death, with favourable profile in the natural history of cardiac channelopathies $(\mathrm{CCh})^{3}$, which are inherited cardiac ion channels disorders associated with potential ventricular arrhythmias and sudden death in the presence of a structurally normal heart ${ }^{4,}{ }^{5}$. The most prevalent CCh are congenital long QT syndrome (LQTS), Brugada syndrome (BrS) and catecholaminergic polymorphic ventricular tachycardia (CPVT), which account for approximately one-third of unexplained sudden deaths ${ }^{6,7}$.

The treatment goal of $\mathrm{CCh}$ is to avoid arrhythmias and sudden death and it remains a challenge. Betablockers for LQTS and CPVT, and quinidine for BrS, have generally been used for therapeutic optimization, 
minimizing repolarization changes and also reducing ventricular arrhythmias. In cases of syncope, torsade de pointes or cardiac arrest requiring cardiopulmonary resuscitation, ICD and/or cardiac sympathetic denervation are often the treatment choices ${ }^{8}$.

With increased awareness of genetic arrhythmogenic disorders, the rate of ICD implantation in young adult population is also increasing. Patients can be submitted to conventional (sub/supra pectoral) ICD implantation or subcostal approach ${ }^{9}$. The vast majority of devices employ bipolar leads, resulting in less susceptibility to electromagnetic interference (EMI) ${ }^{2}$.

Many studies assessed if magnetic electrical and electromagnetic fields from dental devices could affect cardiovascular implantable electronic devices (CIEDs). Electric toothbrush, amalgamator, high- and low-speed handpieces, endodontic heat carriers, electric pulp tester, apex locators, wired curing light unit and piezoelectric unit do not altering pacing function ${ }^{10-13}$. Ultrasonic scalers and ultrasonic cleaning systems could interfere with CIEDs, but those EMI events may not be clinically significant ${ }^{14,}{ }^{15}$. Unipolar electrosurgery units produce electromagnetic disturbances that may possibly affect the function of ICD by delivering an unintentional shock ${ }^{10}$.

However, dental anesthetic management of patients with ICD are limited to case reports ${ }^{16,17}$. These patients demand adequate care and analgesia because of the potential risk of life-threatening events (LTE) such as sustained ventricular tachycardias, ICD therapies during the intervention and arrhythmic syncope ${ }^{18}$. It is crucial to provide a wary dental treatment environment averting triggers for arrhythmic events, such as emotional stress, auditory stimuli or increased vagal tone ${ }^{19,20}$. Dentists should obtain a detailed medical history of patients with ICD and preferably with a cardiologist background ${ }^{21}$.

It is important to emphasize that the use of local dental anesthesia in ICD recipients requires basic knowledge in order to avoid eminent complications in patients with risk of sudden cardiac death. In our previous study ${ }^{22}$, the use of local dental anesthesia with and without epinephrine in selected stable patients with LQTS and BrS did not result in life-threatening arrhythmias, though the maximum heart rate increased after the use of vasoconstrictor during the anesthesia period. We decided to make a subanalysis in cases with CCh and ICD recipients.

The aim of our study was to ascertain the safety of lidocaine $2 \%$ with and without epinephrine 1:100,000 in patients with CCh and ICD.

\section{METHODS}

\section{Population}

This study comprised consecutive patients treated at the Heart Institute of Hospital das Clínicas, Faculdade de Medicina, Universidade de São Paulo with inherited CChs (LQTS, BrS or CPVT) and ICD receiving optimal drug therapy. The inclusion criteria were patients having dental caries, unsatisfactory restorations in the mandible and restorative dental treatment indication. The exclusion criteria were the following: allergy to lidocaine, sodium metabisulfite, or methylparaben; patients with recurrent syncope or sustained arrhythmias documented for at least 3 months, including appropriate and innappropriate ICD shocks; patients who had received epinephrine in the previous 24 hours; and patients with a body weight $<20 \mathrm{~kg}$ (a child [?] 6 years old because of the maximum safe dose of lidocaine, $4.4 \mathrm{mg} / \mathrm{kg}$, used in 2 anesthetic cartridges ${ }^{23}$ ).

All patients were included after reading and signing the written informed consent form. This study was approved by the Ethics Committee of Hospital das Clinicas HCFMUSP, Faculdade de Medicina, Universidade de Sao Paulo (18221913.5.0000.0068) and was previously registered at ClinicalTrials.gov (ID: NCT03182777) and can be accessed at https: // clinicaltrials. gov/ ct2/ show/ NCT03182777? term= NCT031827778 rank= 1

\section{Monitoring}

We compared the use of a mandibular nerve block with 2 cartridges $(3.6 \mathrm{~mL})$ of $2 \%$ lidocaine $(72000 \mu \mathrm{g}$ of lidocaine) without a vasoconstrictor and 2 cartridges of $2 \%$ lidocaine with 1:100 000 epinephrine (36 $\mu \mathrm{g}$ of 
epinephrine) in all patients resulting in 2 conditions, verifying the occurrence of life-threatening arrhythmias (hemodynamically unstable arrhythmias, sustained ventricular tachycardia, or appropriate device shocks) in selected patients with CChs and ICD.

All patients were submitted to two sessions of restorative dental treatment with a washout period of 7 days (crossover trial) and the same patients were further used as their own controls.

The procedure was blinded to the patient and the dentist performing to the presence or absence of epinephrine, then the carpule syringe was covered with sterile aluminum foil by one of our research team members. Our research team developed a randomization program in Excel (Microsoft Office) accomplished by the randomization of the anesthetic solution application.

The cardiac electrical activity was registred and analyzed during the two sessions in all patients for 28 hours by a Holter monitor (SEER Light Extend; GE Healthcare Brazil) with 3-channels (V1, V3, and V5 equivalent leads), starting 1 hour before the procedure. The occurrence and frequency of ventricular and supraventricular arrhythmias, identified on a minute-by-minute basis over the 28-hour study period (basal period, anesthesia period, procedure period, and postprocedure period) and the minimum, medium, and maximum heart rates (HRs) were included as electrocardiographic variables studied.

Specific records were also conducted at 3 time points during the dental treatment (at the beginning of the basal period, 15 minutes after the anesthesia application, and at the end of the procedure) by the 12-lead electrocardiography, digital sphygmomanometry for blood pressure (BP), and assessment of the Facial Image Scale for anxiety.

Corrected QT (QTc) interval in LQTS patients was calculated using Bazett's formula (QTc=QT interval/RR interval), preferably in lead II, or V2 and V5. QTc values $>460 \mathrm{~ms}$ for women were considered abnormal ${ }^{24}$. The QT interval was manually measured from the beginning of the QRS complex to the end of the T wave from all 12 leads using the tangent method. Whenever the end of the T wave could not be determined in any given lead, this lead was excluded from the analysis. The same cardiologist NQSO made all measurements, later confirmed by a second cardiologist FCCD, both blinded to the patients' data. Occasional disagreements were resolved by consensus. Changes in QTc (categorized in $>10 \%$ of shortening or lengthening of QTc) were also analyzed.

For patients with BrS, an additional high right precordial lead was included to observe the possible occurrence of dynamic changes during the phases of the dental procedure.

Possible device shocks or ICD therapies were scheduled to be analyzed in all patients by the medical team, independently of any therapies. We also analyzed the morphological pattern of dynamic changes in the right precordial leads in patients with $\mathrm{BrS}$, as previously described ${ }^{22}$.

\section{Statistical Analysis}

Due to the exploratory nature of this small cohort pilot study, there was no calculation in sample size. It was not possible to estimate the real incidence of arrhythmias with the use of local dental anesthetics in patients with CCh and ICD, since literature is limited by the absence of studies in this population.

All variables were analyzed quantitatively, including the observation of the minimum and maximum measured parameter values and the calculation of means, SDs, and medians. All qualitative variables were calculated with absolute and relative frequencies. For comparison between 2 groups in relation to the means, we used the paired Student's t test. The Wilcoxon signed-rank test was used when the normality assumption was rejected.

Statistical analysis was performed using the SPSS (Statistical Package for the Social Sciences), version 20.0 (SPSS, Inc, Chicago, IL). All tests were two tailed and the level of significance was set at $5 \%$.

\section{RESULTS}


Twenty-four procedures were performed in 12 patients (9 women, 3 men) with CCh and ICD: 7 (58.3\%) had LQTS, $4(33.3 \%)$ had BrS and $1(8.3 \%)$ had CPVT. Ages ranged from 17 and 67, with a mean age of $42.5+$ 14 , and 8 patients $(66.6 \%)$ were white. All patients were in stable condition, with no recent events before the dental care and receiving antiarrhythmic drug treatment (if indicated) according to medical decision (Table 1).

There were no symptoms or ICD therapy (antitachycardia pacing therapy [ATP] and shocks triggered). No complications occurred during the dental procedure requiring interruption. After administration of 2 cartridges of anesthetics, all patients did not complain of pain in both sessions, that lasted from 32 to 93 minutes, with an average of $55+15$ minutes.

Holter monitoring registered the HR and numbers of supraventricular and ventricular premature beats per hour in both conditions (with and without epinephrine) during the study periods, with no significant difference between them $(\mathrm{P}>0.05)$ (Tables 2$)$.

No LTE occurred during dental treatment, regardless of the type of anesthesia. No patient with ICD received device shocks during the procedures and no sustained arrhythmias were observed.

Patients with LQTS and ICD did not show any LTE and the QTc measurements showed no statistically significant differences (Table 3). After administration of anesthesia, changes in QTc (categorized in $>10 \%$ of shortening or lengthening of QTc) occurred in 2 patients, shortening this interval.

The four patients with BrS had no changes in ECG morphology in both conditions, with and without epinephrine, during the studied 3 moments and had no LTE.

The patient with CPVT did not showed occurrence and documentation of ventricular arrhythmia in the electrocardiographic tracings.

At the recording time points, with and without epinephrine, there were no significant differences in systolic and diastolic $\mathrm{BP}$ values and in anxiety measures.

\section{DISCUSSION}

In our protocol study, no patient received ICD therapy (ATP or appropriate/ inappropriate discharges) during the dental treatment under local anesthesia regardless of the use of a vasoconstrictor. No sustained arrhythmias were observed, indicating that stable or treated patients with CCh and ICD can even be sheltered when epinephrine at pattern doses is used with lidocaine.

The safety of these anesthetics could be observed in our study protocol when QTc shortened in 2 LQTS patients, suggesting a possible protective effect of lidocaine. No LTE occurred in patients with LQTS and no significant prolongation of the QT interval were observed.

Patients with BrS preserved the same electrocardiographic pattern during the studied three-time points in both conditions, with and without epinephrine, and any dynamic changes occurred in the high precordial leads.

There was no procedure-related complication in the patient with CPVT, and fortunately with no ventricular arrhythmia documented, even under epinephrine use. These results could also be in part explained by the possible protective effect of lidocaine in both periods, as well by our strict inclusion criteria (only stable patients).

In a previous study, the use of local dental anesthesia with and without epinephrine in selected stable patients with LQTS and BrS did not result in life-threatening arrhythmias, though the maximum heart rate increased after the use of vasoconstrictor during the anesthesia period ${ }^{25}$.

According to American Society of Anesthesiologists Task Force ${ }^{26}$, anesthetic techniques do not influence cardiac rhythm management devices (CRMD) function. However, anesthetic-induced physiologic changes 
(i.e. , cardiac rate, rhythm, or ischemia) in the patient may induce unexpected CRMD responses or adversely affect the CRMD-patient interaction.

Anesthetic drugs have not been demonstrated to affect pacing thresholds, though the physiologic consequences of anesthetic management may. Myocardial ischemia and high blood levels of local anesthetics may increase electrophysiologic thresholds, but one hardly needs to be cautioned in these areas. It is noteworthy the importance to avoid hyperventilation in these patients, which could abruptly lower serum potassium levels ${ }^{2}$.

Vital parameters could be influenced by the use of vasoconstrictors added to the stress of the dental procedure $^{27}$. The findings of the present study did not show significantly changes in BP and anxiety comparing the conditions with and without epinephrine in patients with LQTS, BrS and CPVT.

However, $\mathrm{Tom}^{28}$ pointed out that anesthetics with epinephrine used in dentistry may have considerable effects upon the sensing and function of CIED. They can promote tachyarrhythmias and initiate ICD events, if there is no prior modification of anesthetic techniques and particularly with higher doses.

The insertion of an ICD can be performed under local anesthesia with sedation during induction of VF, testing of the defibrillator, and placement in the subpectoral pocket, thus avoiding general anesthesia. The total dose of local anesthetic should be minimized, and systemic absorption limited by the use of lidocaine with epinephrine. Local anesthetics, because of their sodium channel blockade, may exacerbate Brugada ECG changes. However, the class IB drugs mexilitine and lidocaine have not been shown to cause ST-segment elevation $^{29}$ which, in the final analysis, also suggests a protective and safety effect.

Theodotou and Cillo ${ }^{16}$ described a case report using local anesthetic for dental treatment in 55-year-old patient with ICD, BrS and valvular heart disease. He was subjected to exodontia and abscess drainage under general anesthesia and 15 milligrams of lidocaine with 1:100 000 epinephrine was applied in the intraoral region for local anesthesia of the operated area. The patient had not adverse cardiac events or intraoperative complications.

The dental care of a seven-year-old boy with a medical history of LQTS using ICD was described by Karp, Ganoza $^{30}$. After a syncope episode with development of torsades de pointes, he suffered dental trauma and had no complications in his tooth extraction under general anesthesia.

In our casuistry, it was noted that 5 out of 7 patients with LQTS were carriers of type 2 LQTS, which characteristically could have events triggered by noise and emotions ${ }^{31}$. The dental environment needs to be as calm and quiet as possible, but devices noise is inevitable. Fortunately, none of the patients with LQTS had LTE, provided that the exclusion criteria were respected.

A case report of a 13-year-old CPVT patient was described ${ }^{32}$, whose had already undergone to previous dental treatment under general anesthesia. Due to recurrence of carious lesions and the need for further intervention, the cardiologist did not contraindicate the use of local anesthetic with epinephrine. However, the dentist considered prudent the use $3 \%$ mepivacaine for local anesthesia in the amount of 3 cartridges, besides the administration of nitrous oxide to perform dental restorations in the dental chair in a hospital setting. We also had a favorable experience with one CPVT patient using lidocaine with and without epinephrine.

It is crucial to comprehend the perioperative management of these patients to avoid preventable complications, as the EMI sources should be kept in distance from CIEDs as it is possible ${ }^{2}$ and to be aware of inadvertent local anesthesia intravascular administration ${ }^{33}$. In our protocol we did not use sources that could interfere with sensing and pacing activity.

One of our limitations is the fact that this protocol can be applied only to stable patients, as mentioned in methods. Our small sample of patients also limits strong statistical power of efficacy. However, this protocol can be used for exploratory data for future large studies or meta-analyzes. 
To the authors' knowledge, this is the first study (although small) to investigate the use of local dental anesthesia in consecutive patients with CCh and ICD, without detectable adverse clinical impact.

\section{CONCLUSION}

The use of lidocaine with and without epinephrine in CCh and ICD recipients did not result in life-threatening events and had no clinical impact on patient safety. These preliminary findings need to be confirmed in a larger population with CCh and ICD.

\section{ACKNOWLEDGMENTS \\ SOURCE OF FUNDING}

This research was supported by Fundação de Amparo à Pesquisa do Estado de São Paulo (Foundation for Research Support of São Paulo State) 2013/24283-1.

\section{Author contributions}

Oliveira, A: Contributed to conception, design, data acquisition, analysis, and interpretation, drafted and critically revised the manuscript.

Neves, I: Contributed to conception, design, data analysis and interpretation, drafted and critically revised the manuscript.

Sacilotto, L: Contributed to conception, data acquisition, analysis and interpretation, drafted and critically revised manuscript.

Olivetti, N: Contributed to conception, data analysis and interpretation, drafted and critically revised manuscript.

Bueno, S: Contributed to conception, data analysis and interpretation, drafted and critically revised manuscript.

Pessente, G: Contributed to conception, data acquisition and analysis, and critically revised manuscript.

Paul, M: Contributed to conception, design, data acquisition, and critically revised manuscript.

Montano, T: Contributed to conception, design, data interpretation, and critically revised manuscript.

Carvalho, C: Contributed to conception, data acquisition, and critically revised manuscript.

Grupi, C: Contributed to conception, design, data analysis, and critically revised manuscript.

Barbosa, S: Contributed to conception, design, data acquisition, analysis, and interpretation, and critically revised manuscript.

Pastore, C: Contributed to conception, design, data acquisition and analysis, and critically revised manuscript.

Samesima, N: Contributed to design, data analysis, and critically revised manuscript.

$\mathrm{Wu}, \mathrm{T}$ : Contributed to conception, data analysis, and critically revised manuscript.

Hachul, D: Contributed to design, data analysis and critically revised manuscript.

Scanavacca, M: Contributed to conception, design, data interpretation, and critically revised manuscript.

Neves, R: Contributed to conception, design, data acquisition, analysis, and interpretation, and critically revised manuscript.

Darrieux, F: Contributed to conception, design, data acquisition, analysis, and interpretation, drafted and critically revised manuscript.

All authors gave their final approval and agree to be accountable for all aspects of the work. 


\section{REFERENCES}

1. Rapsang AG, Bhattacharyya P. Pacemakers and implantable cardioverter defibrillators-general and anesthetic considerations. Braz J Anesthesiol 2014;64(3):205-14.

2. Stone ME, Apinis A. Current perioperative management of the patient with a cardiac rhythm management device. Semin Cardiothorac Vasc Anesth 2009;13(1):31-43.

3. Priori SG, Napolitano C, Gasparini M, et al. Natural history of Brugada syndrome: insights for risk stratification and management. Circulation 2002;105(11):1342-7.

4. Mizusawa Y. Recent advances in genetic testing and counseling for inherited arrhythmias. J Arrhythm 2016;32(5):389-97.

5. Cerrone M, Priori SG. Genetics of sudden death: focus on inherited channelopathies. Eur Heart J 2011;32(17):2109-18.

6. Semsarian C, Ingles J. Molecular autopsy in victims of inherited arrhythmias. J Arrhythm 2016;32(5):35965.

7. Kauferstein S, Kiehne N, Neumann T, Pitschner HF, Bratzke H. Cardiac gene defects can cause sudden cardiac death in young people. Dtsch Arztebl Int 2009;106(4):41-7.

8. Tomaselli GF, Barth AS. Ion Channel Diseases: an Update for 2016. Curr Treat Options Cardiovasc Med $2016 ; 18(3): 21$.

9. Irfan G, Czapla J, Saitoh Y, et al. Implantable cardioverter defibrillator therapy in young individuals: comparison of conventional and subcostal approaches-a single-centre experience. Europace 2017;19(1):81-87.

10. Maheshwari KR, Nikdel K, Guillaume G, et al. Evaluating the effects of different dental devices on implantable cardioverter defibrillators. J Endod 2015;41(5):692-5.

11. Dadalti MT, da Cunha AJ, de Araujo MC, de Moraes LG, Risso Pde A. Electromagnetic interference of endodontic equipments with cardiovascular implantable electronic device. J Dent 2016;46:68-72.

12. Gomez G, Jara F, Sanchez B, et al. Safety concerns of Piezoelectric Units in Implantable Cardioverter Defibrillator. J Oral Maxillofac Surg 2018;76(2):273-77.

13. Dadalti MTS, da Cunha A, Araujo MCP, Moraes LGB, Risso PA. Electromagnetic interference of dental equipment with implantable cardioverter defibrillators. Acta Odontol Scand 2017;75(8):584-87.

14. Elayi CS, Lusher S, Meeks Nyquist JL, et al. Interference between dental electrical devices and pacemakers or defibrillators: results from a prospective clinical study. J Am Dent Assoc 2015;146(2):121-8.

15. Roedig JJ, Shah J, Elayi CS, Miller CS. Interference of cardiac pacemaker and implantable cardioverterdefibrillator activity during electronic dental device use. J Am Dent Assoc 2010;141(5):521-6.

16. Theodotou N, Cillo JE, Jr. Brugada syndrome (sudden unexpected death syndrome): perioperative and anesthetic management in oral and maxillofacial surgery. J Oral Maxillofac Surg 2009;67(9):2021-5.

17. Shah AH, Khalil HS, Kola MZ. Dental management of a patient fitted with subcutaneous Implantable Cardioverter Defibrillator device and concomitant warfarin treatment. Saudi Dent J 2015;27(3):165-70.

18. Liu C, Zhao L, Tang H, et al. Life-threatening false alarm rejection in ICU: using the rule-based and multi-channel information fusion method. Physiol Meas 2016;37(8):1298-312.

19. Schimpf R, Veltmann C, Wolpert C, Borggrefe M. Channelopathies: Brugada syndrome, long QT syndrome, short QT syndrome, and CPVT. Herz 2009;34(4):281-8.

20. Rochford C, Seldin RD. Review and management of the dental patient with Long QT syndrome (LQTS). Anesth Prog 2009;56(2):42-8. 
21. Jowett NI, Cabot LB. Patients with cardiac disease: considerations for the dental practitioner. Br Dent J 2000;189(6):297-302.

22. Oliveira ACG, Neves I, Scanavacca M, et al. Arrhythmic Events in Patients With Cardiac Channelopathies Submitted to Local Dental Anesthesia. A Randomized Pilot Study: Am Heart Assoc; 2019.

23. Council on Clinical Affairs AAoPD. Guideline on Use of Local Anesthesia for Pediatric Dental Patients. Pediatr Dent 2015;37(5):71-7.

24. Postema PG, Wilde AA. The measurement of the QT interval. Curr Cardiol Rev 2014;10(3):287-94.

25. Oliveira ACG, Neves ILI, Sacilotto L, et al. Is It Safe for Patients With Cardiac Channelopathies to Undergo Routine Dental Care? Experience From a Single-Center Study. J Am Heart Assoc 2019;8(15):e012361.

26. Practice advisory for the perioperative management of patients with cardiac rhythm management devices: pacemakers and implantable cardioverter-defibrillators: a report by the American Society of Anesthesiologists Task Force on Perioperative Management of Patients with Cardiac Rhythm Management Devices. Anesthesiology 2005;103(1):186-98.

27. Takahashi Y, Nakano M, Sano K, Kanri T. The effects of epinephrine in local anesthetics on plasma catecholamine and hemodynamic responses. Odontology 2005;93(1):72-9.

28. Tom J. Management of Patients With Cardiovascular Implantable Electronic Devices in Dental, Oral, and Maxillofacial Surgery. Anesth Prog 2016;63(2):95-104.

29. Cordery R, Lambiase P, Lowe M, Ashley E. Brugada syndrome and anesthetic management. J Cardiothorac Vasc Anesth 2006;20(3):407-13.

30. Karp JM, Ganoza GG. Dental trauma after cardiac syncope in a patient with long QT syndrome. Pediatr Dent 2006;28(6):547-52.

31. Meyer JS, Mehdirad A, Salem BI, Kulikowska A, Kulikowski P. Sudden arrhythmia death syndrome: importance of the long QT syndrome. Am Fam Physician 2003;68(3):483-8.

32. Olufunke Adewumi A, Grace Tucker L. Dental management of a patient with catecholaminergic polymorphic ventricular tachycardia: a case report. J Dent Child (Chic) 2013;80(2):101-4.

33. Rood JP. Adverse reaction to dental local anaesthetic injection-'allergy' is not the cause. Br Dent J 2000;189(7):380-4.

\section{TABLES}

Table 1. Data from ICD recipients with CCh

\begin{tabular}{lllllllll}
\hline CCh & Patient random number & Sex & Age & ICD Prevention & Symptoms & FH & Gene variants & ICD \\
\hline LQTS & 12 & F & 67 & secondary & ACA & yes & KCNH2 & Luma \\
& 21 & F & 41 & secondary & ACA & no & KCNH2 & Luma \\
& 22 & F & 36 & primary & asymptomatic & yes & KCNQ1 & Linox \\
& 23 & M & 28 & primary & syncope & no & KCNH2 & Luma \\
& 26 & M & 17 & secondary & ACA & no & KCNH2 & Analy \\
& 27 & F & 53 & secondary & ACA & yes & NI & Fortify \\
& 29 & F & 41 & secondary & ACA & yes & KCNH2 & Protec \\
BrS & 5 & M & 51 & secondary & ACA & no & NI & Fortify \\
& 11 & M & 39 & primary & syncope & yes & NI & Fortify \\
& 13 & F & 62 & primary & palpitations & yes & NI & Dynag \\
& 15 & M & 41 & primary & palpitations & yes & NI & Virtuc \\
CPVT & 16 & F & 34 & secondary & ACA & no & NI & Protec \\
\hline
\end{tabular}


Legend: CCh - cardiac channelopathy

Sex - M: male / F: female

ICD - implantable cardioverter defibrillator

ACA - Aborted cardiac arrest

SCD - sudden cardiac death

FH - Family history (SCD or channelopathy)

NI - not identified

BT - Biotronik

SJ - ST Jude/ Abbott

MD - Medtronic

BS - Boston Scientific

Table 2. Medium Heart Rate, density of ventricular arrhythmias and ICD therapy of the sample during study periods

\begin{tabular}{|c|c|c|c|c|}
\hline & & $\begin{array}{l}\text { Without } \\
\text { Epinephrine }\end{array}$ & $\begin{array}{l}\text { With } \\
\text { Epinephrine }\end{array}$ & P Value \\
\hline \multirow[t]{10}{*}{ 28h Period } & $\begin{array}{l}\text { medium HR } \\
\text { (bpm) }\end{array}$ & & & 0.401 \\
\hline & mean $\pm \mathrm{SD}$ & $81.4 \pm 9.2$ & $80.3 \pm 8$ & \\
\hline & $\begin{array}{l}\text { median (min.; } \\
\max .)\end{array}$ & $80(68 ; 98)$ & $80.5(67 ; 92)$ & \\
\hline & VPB & & & $0.541^{*}$ \\
\hline & mean $\pm \mathrm{SD}$ & $11.73 \pm 27,79$ & $37.36 \pm 113.83$ & \\
\hline & $\begin{array}{l}\text { median (min.; } \\
\text { max.) }\end{array}$ & $0.1(0 ; 96,7)$ & $0.1(0 ; 397,8)$ & \\
\hline & NSVT & & & $0.655^{*}$ \\
\hline & mean $\pm \mathrm{SD}$ & $0.25 \pm 0.87$ & $7.83 \pm 27.14$ & \\
\hline & $\begin{array}{l}\operatorname{median}(\min ; \\
\max .)\end{array}$ & $0(0 ; 3)$ & $0(0 ; 94)$ & \\
\hline & ICD therapy & 0 & 0 & \\
\hline \multirow[t]{10}{*}{ Basal Period } & $\begin{array}{l}\text { medium HR } \\
\text { (bpm) }\end{array}$ & & & 0.645 \\
\hline & mean $\pm \mathrm{SD}$ & $76.1 \pm 8.3$ & $76.6 \pm 6.9$ & \\
\hline & $\begin{array}{l}\text { median (min.; } \\
\text { max.) }\end{array}$ & $73.5(65 ; 93)$ & $75,5(66 ; 91)$ & \\
\hline & VPB & & & $0.752^{*}$ \\
\hline & mean $\pm \mathrm{SD}$ & $9.58 \pm 28.27$ & $65.42 \pm 199.09$ & \\
\hline & $\begin{array}{l}\text { median (min.; } \\
\text { max.) }\end{array}$ & $0(0 ; 98)$ & $0,5(0 ; 694)$ & \\
\hline & NSVT & & & $0.317^{*}$ \\
\hline & mean $\pm \mathrm{SD}$ & $0 \pm 0$ & $1.33 \pm 4.62$ & \\
\hline & $\begin{array}{l}\text { median (min.; } \\
\text { max.) }\end{array}$ & $0(0 ; 0)$ & $0(0 ; 16)$ & \\
\hline & ICD therapy & 0 & 0 & \\
\hline Anesthesia & medium HR & & & 0.261 \\
\hline Period & $(\mathrm{bpm})$ & & & \\
\hline
\end{tabular}




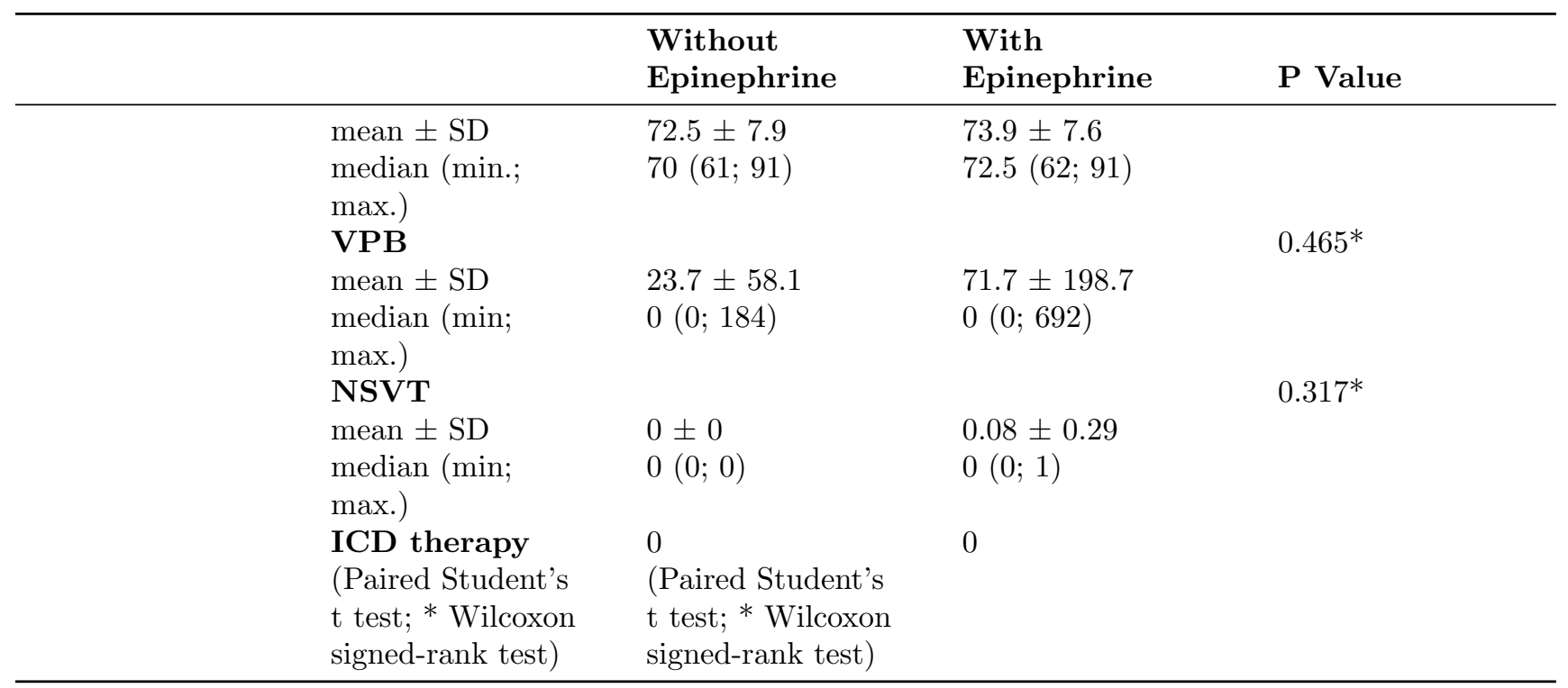

Legend: VPB - ventricular premature beats

NSVT - number of non-sustained ventricular tachycardia

Table 3. Mean, standard deviation, median, minimum and maximum QTc and average QTc at three study moments in the conditions without vasoconstrictor and with epinephrine in LQTS patients

\begin{tabular}{llll}
\hline & Without vasoconstrictor & With epinephrine & p \\
\hline $\begin{array}{l}\text { Basal period } \\
\text { QTc }\end{array}$ & & & 0.911 \\
mean \pm SD & $482.1 \pm 42.7$ & $484.4 \pm 28.1$ & \\
median (min.; max.) & $490(412 ; 542)$ & $477(447 ; 521)$ & \\
End of anesthesia & & & 0.053 \\
QTc & & $478 \pm 23.4$ & \\
mean \pm SD & $456 \pm 25.5$ & $480(447 ; 519)$ & \\
median (min.; max.) & $468(420 ; 480)$ & & 0.306 \\
End of procedure & & $473.7 \pm 33$ & \\
QTc & & $456(440 ; 530)$ & 0.362 \\
mean \pm SD & $461.9 \pm 34.6$ & & \\
median (min.; max.) & $468(420 ; 524)$ & $478.7 \pm 25.8$ & \\
$\begin{array}{l}\text { Average QTc } \\
\text { mean } \pm \text { SD }\end{array}$ & $466.7 \pm 30.8$ & $471.7(447 ; 523.3)$ & \\
median (min.; max.) & $476(417.3 ; 506)$ & &
\end{tabular}

\section{FIGURE LEGENDS}

Figure 1. Electrocardiographic tracing of a patient with LQTS

A) At the basal period, presenting QTc $=495 \mathrm{~ms}$ 


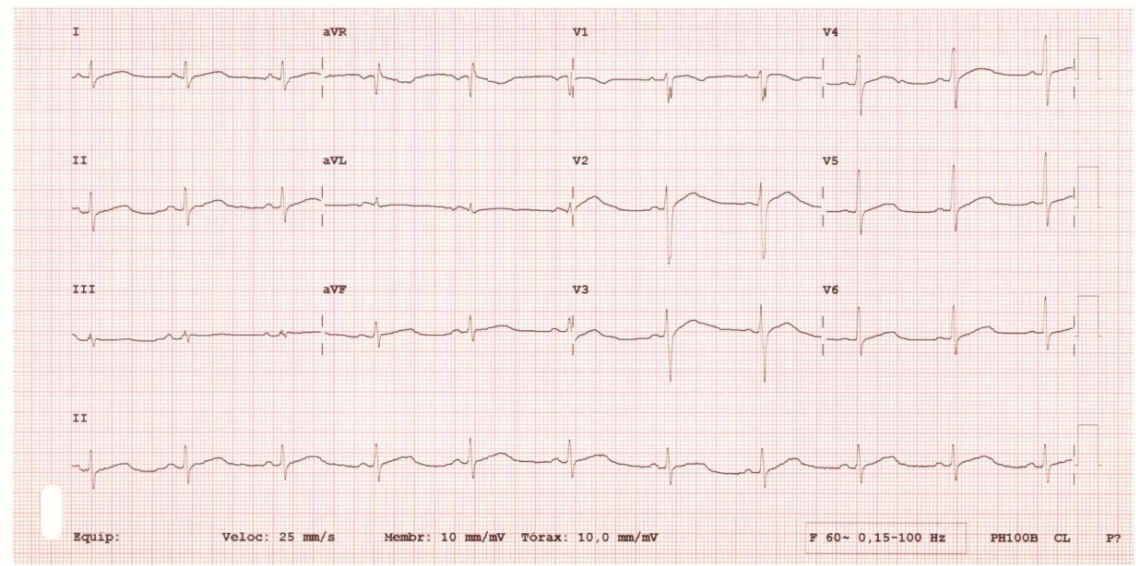

B) At the end of anesthesia, with epinephrine, presenting QTc $=480 \mathrm{~ms}$

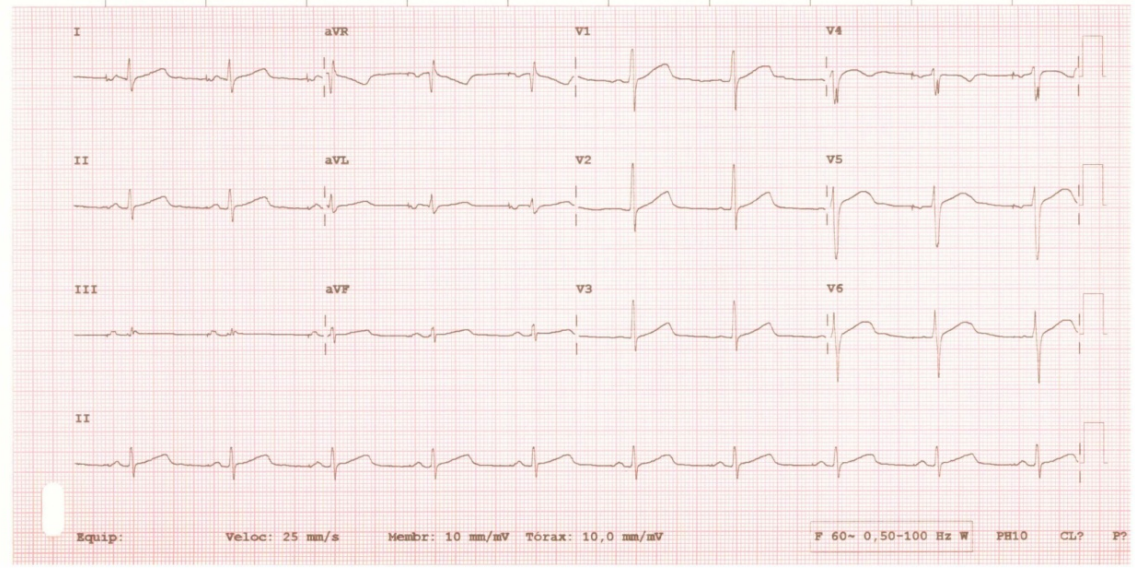

C) At the end of procedure, with epinephrine, presenting QTc $=440 \mathrm{~ms}$

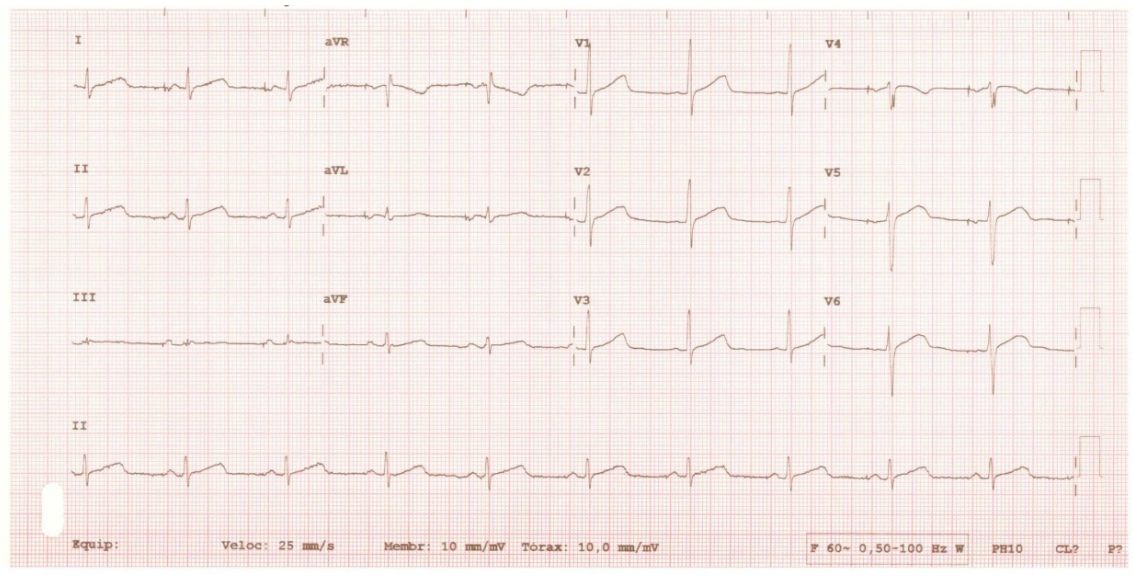

